\title{
Why Impacted Yarns Break at Lower Speed than Classical Theory
}

\author{
Predicts \\ James D. Walker* and Sidney Chocron \\ Southwest Research Institute, San Antonio, Texas, 78238, USA \\ *corresponding author: james.walker@swri.org
}

This paper has also been submitted in its entirety for the JAM publication in conjunction with ISB 26.

\begin{abstract}
Fabrics are an extremely important element of body armors and other armors. Understanding fabrics requires understanding how yarns deform. Classical theory has shown very good agreement with the deformation of a single yarn when impacted. However, the speed at which a yarn would break based on this classical theory is not correct; it has been experimentally noted that yarns break when impacted at a lower velocity. This paper explores the mechanism of yarn breakage. The problem of the strike of a yarn by a flat-faced projectile is analytically solved for early times. It is rigorously demonstrated that when a flat-faced projectile strikes a yarn, the breaking speed will always be at least $11 \%$ less than the classical-theory result. It is further shown that when the yarn material in front of the projectile is "bounced" off the front surface due to the impact, that the breaking speed is further reduced. If the yarn bounces elastically off the surface due to the impact at twice the impact velocity (the theoretical maximum), there is a $40 \%$ reduction in the breaking speed of a yarn.
\end{abstract}

A beautiful theory of yarn deformation under an applied boundary condition was developed by Smith [1,2]. This theory was developed in the context of impact on yarns. There has been extensive experimental work showing that the wave propagation speeds of the longitudinal and transverse waves and the subsequent deflections are well modeled by the theory. However, numerous researchers have realized that the yarn breaking speed inferred from the classical theory is larger than what is observed in experiment.

Why are the impacted yarns breaking at lower speeds than the theory predicts? Most often yarns are impacted by fragment simulating projectiles or right circular cylinders. These projectiles have flat impact faces. In our computational investigation of this phenomenon, we have noticed that the flat face impact leads to a "bounce" of the yarn off the impact surface (Fig. 1). A longitudinal and transverse wave are produced in the yarn, originating at the impactor edges, traveling both outward away from the impactor and inward where the waves from opposing impactor edges. The waves meet at the geometric center of the yarn in front of the projectile; the stress and strain in the yarn double, leading to higher stresses. After this first wave effect, we also see, during more complicated wave interactions a small increase in stress until the material is relieved to the classical (Smith) solution stress at later time. 


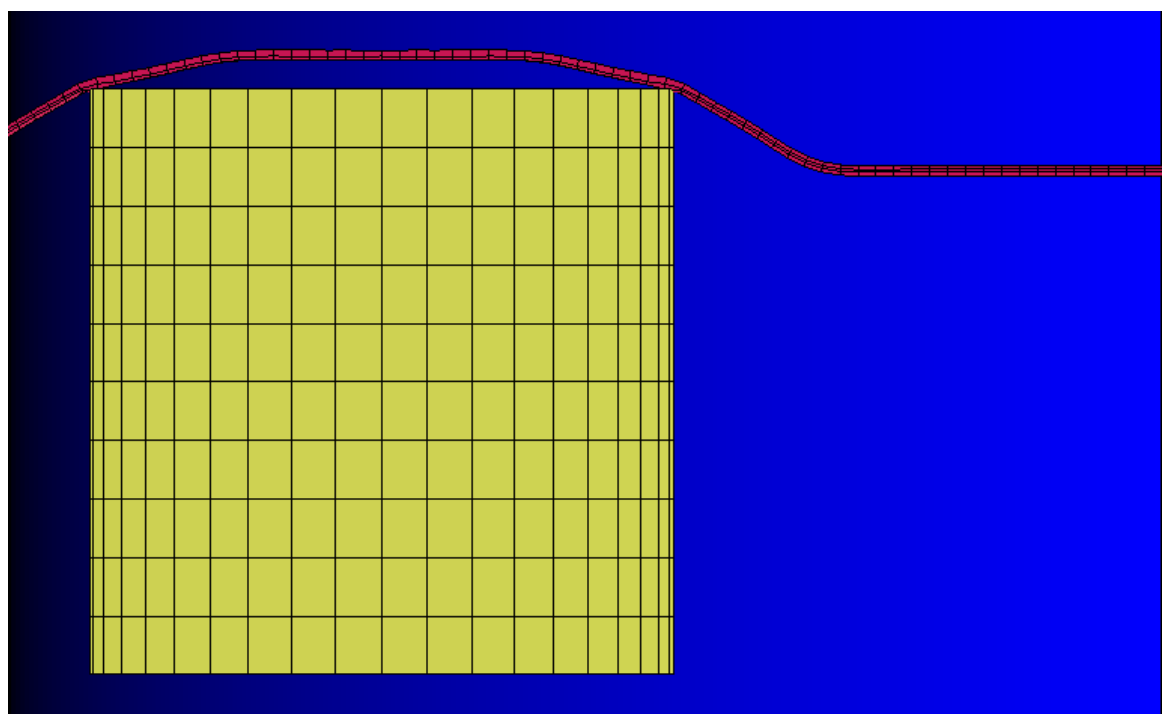

Figure 1. Right circular cylinder flat-faced impactor (moving up) striking a yarn, from a LS-DYNA computation. The bends in the yarn show the transverse waves emanating from the edges of the impactor. The upward motion of the yarn away from the impactor face is due to the impact.

In this paper we analytically investigate the possibility that the bounce could be the cause of the reduced breaking speed of the yarn. As part of the investigation, we have rigorously shown the surprising result that when a flat-faced projectile strikes a yarn, the breaking speed will always be at least 11\% less than the classical-theory result. We show that there is a further reduction if there is bounce of the yarn in front of the projectile face. Theoretically the bounce can at most lead to the yarn having twice the velocity of the projectile - i.e., the yarn moves away from the projectile face at again the projectile velocity. The theoretical upper bound bounce result is based on an elastic material being struck by a massive a rigid surface. When the bounce of the yarn is twice the impacting velocity, there is a $40 \%$ reduction in the breaking speed of a yarn. This bounce has been experimentally observed by Field and Sun with a nylon sphere striking a rubber band [3]. Though those results are for a sphere, the top of the sphere is reasonably flat enough that the present theory applies.

This paper presents the simple theory but from a new point of view as suggested by Walker's work on fabrics [4-5]. Then the impact of a yarn by a flat-faced projectile is solved. These results described in the previous paragraph are shown and discussed. Thus, the reason the yarn breaks is due to the finite front face extent of the projectile which leads to waves traveling from the edge of the projectile to interact at their center. There is more reduction by the impacting placing in motion the yarn material directly in front of the impactor by the impact. Figure 2 shows a comparison of these results on the breaking speed normalized by the classical result of the breaking speed over the range of bounce velocities. The decrease in breaking speed is evident, and is similar for three failure strains considered. 


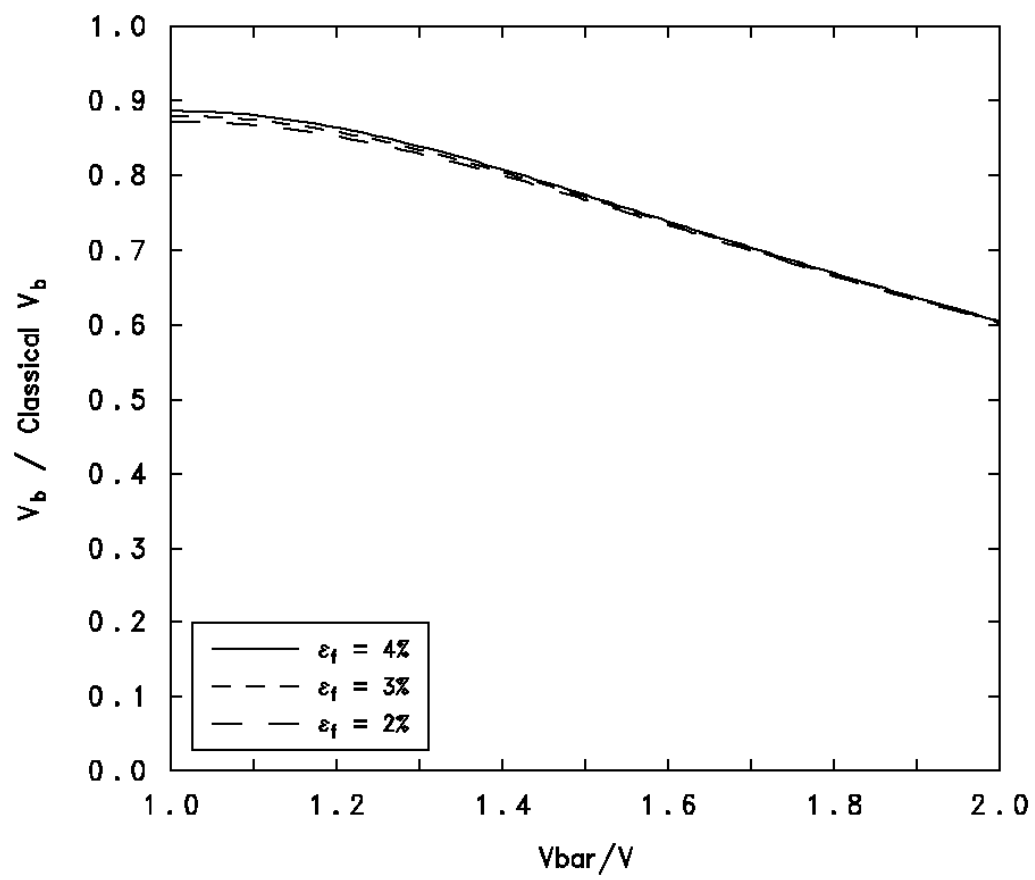

Figure 2. The impacted yarn breaking speed normalized by the breaking speed from the classical theory as a function of the amount of bounce $\bar{V} / V$ due to the impact.

\section{References}

1. J. C. Smith, F. L. McCrackin and H. F. Schiefer. Stress-strain relationships in yarns subjected to rapid impact loading, Part V: Wave propagation in long textile yarns impacted transversely. Textile Research Journal, 28(4):288-302, 1958.

2. P. M. Cunniff. An Analysis of the System Effects of Woven Fabrics Under Ballistic Impact. Textile Research Journal, 62(9):495-509, 1992.

3. J. E. Field and Q. Sun. A high speed photographic study of impact on fibres and woven fabrics. In SPIE Vol. 1358, $19^{\text {th }}$ International Congress on High-Speed Photography and Photonics, 1990.

4. J. D. Walker. Constitutive model for fabrics with explicit static solution and ballistic limit. Proc. 18th Int. Symp. on Ballistics, San Antonio, Texas, 1231-1238, 1999.

5. J. D. Walker. Analytic expression for the deformation and ballistic limit of fabrics. Int. Conf. on Composite Materials, San Diego, CA, SME Technical Paper Em03-336, 2003. 